\title{
Association of BAFF/BLyS overexpression and altered $B$ cell differentiation with Sjögren's syndrome
}

\author{
Joanna Groom, ${ }^{1}$ Susan L. Kalled, ${ }^{2}$ Anne H. Cutler, ${ }^{2}$ Carl Olson, ${ }^{2}$ Stephen A. Woodcock, ${ }^{2}$ \\ Pascal Schneider, ${ }^{3}$ Jurg Tschopp, ${ }^{3}$ Teresa G. Cachero, ${ }^{2}$ Marcel Batten, ${ }^{1}$ Julie Wheway, ${ }^{1}$ \\ Davide Mauri, ${ }^{4}$ Dana Cavill, ${ }^{5}$ Tom P. Gordon, ${ }^{4}$ Charles R. Mackay, ${ }^{1}$ and Fabienne Mackay ${ }^{1}$ \\ ${ }^{1}$ The Garvan Institute of Medical Research, Department of Arthritis and Inflammation, Darlinghurst, Australia \\ ${ }^{2}$ Departments of Immunology, Inflammation, Cell Biology, and Protein Engineering, Biogen Inc., Cambridge, Massachusetts, USA \\ ${ }^{3}$ Institute of Biochemistry, University of Lausanne, Epalinges, Switzerland \\ ${ }^{4}$ Apotech Biochemicals, Epalinges, Switzerland \\ ${ }^{5}$ Department of Immunology, Arthritis and Allergy, Flinders Medical Centre, Bedford Park, Australia
}

Address correspondence to: Fabienne Mackay, The Garvan Institute of Medical Research, 384 Victoria Street, Darlinghurst, NSW 2010, Australia. Phone: 61-2-92958414; Fax: 61-2-92958404; E-mail: f.mackay@garvan.unsw.edu.au.

Received for publication August 31, 2001, and accepted in revised form November 19, 2001.

BAFF (BLyS, TALL-1, THANK, zTNF4) is a member of the TNF superfamily that specifically regulates $B$ lymphocyte proliferation and survival. Mice transgenic $(\mathrm{Tg})$ for BAFF develop an autoimmune condition similar to systemic lupus erythematosus. We now demonstrate that BAFF Tg mice, as they age, develop a secondary pathology reminiscent of Sjögren's syndrome (SS), which is manifested by severe sialadenitis, decreased saliva production, and destruction of submaxillary glands. In humans, SS also correlates with elevated levels of circulating BAFF, as well as a dramatic upregulation of BAFF expression in inflamed salivary glands. A likely explanation for disease in BAFF Tg mice is excessive survival signals to autoreactive $B$ cells, possibly as they pass through a critical tolerance checkpoint while maturing in the spleen. The marginal zone (MZ) B cell compartment, one of the enlarged B cell subsets in the spleen of BAFF Tg mice, is a potential reservoir of autoreactive B cells. Interestingly, B cells with an MZ-like phenotype infiltrate the salivary glands of BAFF $\mathrm{Tg}$ mice, suggesting that cells of this compartment potentially participate in tissue damage in SS and possibly other autoimmune diseases. We conclude that altered B cell differentiation and tolerance induced by excess BAFF may be central to SS pathogenesis.

J. Clin. Invest. 109:59-68 (2002). DOI:10.1172/JCI200214121.

\section{Introduction}

Sjögren's syndrome (SS) is a chronic inflammatory disorder characterized by the malfunction of exocrine glands such as salivary and lacrimal glands, leading to symptoms of dry mouth (xerostomia) and eyes (keratoconjunctivitis sicca) (1). SS is regarded as an autoimmune disease characterized by the presence of large mononuclear cell infiltrates in exocrine glands, B cell hyperreactivity, and various serum autoantibodies (1, 2). SS can develop alone or in association with other autoimmune disorders such as systemic lupus erythematosus (SLE) and rheumatoid arthritis (RA) $(1,2)$.

Abnormal B cell activity is a predominant feature of SS, which is manifested by massive polyclonal B cell activation and elevated secretion of autoantibodies such as rheumatoid factors, anti-Ro (SS-A), anti-La (SS-B), and anti- $\alpha$-fodrin autoantibodies (1-4). Intense $B$ cell activity such as germinal center formation occurs in exocrine glands of some patients, placing them in a high risk category for the development of lymphomas $(1,5)$. However, the role of $B$ cells and autoantibodies in the pathogenesis of SS remains unclear.

BAFF (BLyS, TALL-1, THANK, zTNF4) is a very powerful modulator of B cell biology, expressed by mono- cytes/macrophages and dendritic cells (6-10). BAFF can bind three separate receptors: BCMA (B cell maturation antigen), TACI (transmembrane activator and CAMLinteractor), and BAFF-R (7-9, 11-14). Expression of BCMA and BAFF-R is B cell-specific $(9,14)$, whereas TACI can be found on B cells and on subsets of activated T cells (15). Moreover, BAFF shares two receptors - TACI and BCMA - with another TNF-like ligand named APRIL, which is involved in tumor cell growth (7). Treatment of mice with TACI-Ig fusion protein inhibited $\mathrm{T}$ cell-dependent and -independent immune responses (16) and abolished germinal center formation (17). Treatment of normal mice with soluble BCMA-Ig led to a marked reduction in $B$ cell numbers in the periphery, suggesting a role for BAFF in peripheral B cell homeostasis (18). Increased BAFF-mediated B cell survival has also been shown to enhance humoral immune responses (19). Recently, we showed that BAFF promotes B cell survival in vitro and in vivo, with a predominant role in the spleen on maturing transitional type II (T2) B cells (20). $\mathrm{BAFF}$ can also promote $\mathrm{B}$ cell survival and maturation in vitro (20). Moreover, B cell maturation in BAFF-deficient mice is arrested at the transitional type I (T1) immature B cell stage, which precedes the T2 B cell stage $(21,22)$. 
Overproduction of BAFF is associated with the development of certain autoimmune diseases. BAFF transgenic ( $\mathrm{Tg}$ ) mice have an elevated number of B cells in the periphery, secrete various autoantibodies, and develop an SLE-like condition $(8,9,23)$. Moreover, in BAFF Tg mice, both the $\mathrm{T} 2$ and the marginal zone $(\mathrm{MZ}) \mathrm{B}$ cell subsets are enlarged, suggesting that $\mathrm{T} 2 \mathrm{~B}$ cells may differentiate directly into $\mathrm{MZ} \mathrm{B}$ cells in some circumstances $(24,25)$. Excess BAFF-mediated survival signals might compromise the ability of autoreactive B cells to respond to censoring death signals; therefore, abnormal BAFF production may be a key event in autoimmunity. Interestingly, serum levels of BAFF are significantly higher in patients with SLE and RA than in healthy individuals, suggesting a possible critical role for BAFF in human immune-based rheumatic diseases $(26,27)$. Moreover, treatment with TACI-Ig, used as a decoy receptor, significantly reduced symptoms and even prevented disease in mouse models of SLE and collageninduced arthritis, respectively $(22,28-30)$.

In this report, we describe findings that, as they age, BAFF Tg mice develop a condition that is secondary to their lupus-like disease and has interesting similarities to SS in humans. We also identified a new and potentially pathogenic B cell population with $\mathrm{MZ}$-like features, infiltrating salivary glands of BAFF Tg mice. In an effort to investigate whether dysregulated expression of BAFF could be associated with human SS, we tested sera from patients with SS, and demonstrated levels of circulating BAFF that were significantly elevated compared with levels in healthy control individuals. Both BAFF levels and the incidence of patients producing high levels of BAFF were greater in the SS cohort than in the SLE and RA cohorts. This suggests a more dominant role for BAFF in the progression of SS than in other immune-based rheumatic diseases, which may be related to the intense B cell activity associated with SS.

\section{Methods}

Mice and reagents. Full-length murine BAFF was expressed in Tg mice using the liver-specific $\alpha 1$ antitrypsin promoter with the apoE enhancer, as previously described (23). C57BL/6 mice were purchased from Animal Resource Center (Perth, Australia). BAFF Tg mice were maintained as heterozygotes for the transgene by backcrossing onto C57BL/6 mice. BAFF Tg mice were screened for the presence of the transgene by both PCR and Southern blot analysis using genomic DNA isolated from tail snips 2-3 mm long (23). We used mice from two separate lines of BAFF $\mathrm{Tg}$ mice issued after 10-12 backcrossings onto C57BL/ 6 mice. The phenotype of BAFF Tg mice was not altered while mice were backcrossed onto the C57BL/ 6 background. Age-matched transgene-negative littermates were used as controls. Animals 8-17 months of age were used. Animals were housed under conventional barrier protection and handled in accordance with the Animal Experimentation and Ethics Committee of St. Vin- cent's Hospital (Sydney, Australia), which complies with the Australian code of practice for the care and use of animals for scientific purposes. Flag-tagged soluble human BAFF (amino acids 83-285) was expressed by E. coli and purified as described previously (31). Antihuman BAFF antibodies Buffy-2 (rat IgM), Buffy-5 (rat IgG1), and A21G3.3 (mouse IgG1) were obtained after immunization of rats or mice with recombinant soluble human BAFF as previously detailed (31). These antibodies recognize the TNF homology domain of soluble BAFF. The antibody A21G3.3 was purified as follows: $500 \mathrm{ml}$ of medium from hybridoma cultures was diluted 1:1 with $0.1 \mathrm{M}$ sodium phosphate $(\mathrm{pH} 7.2)$ buffer containing $150 \mathrm{mM} \mathrm{NaCl}$. The diluted media was loaded onto a protein L column (CLONTECH Laboratories Inc., Palo Alto, California, USA) at $1 \mathrm{ml} / \mathrm{min}$ and eluted with $0.1 \mathrm{M}$ glycine ( $\mathrm{pH}$ 2.8). The eluted solution was neutralized with $1 \mathrm{M}$ sodium phosphate buffer ( $\mathrm{pH}$ 7.2). The peak fractions were confirmed by SDS-PAGE. Centricon Plus-20 (Millipore Corp., Bedford, Massachusetts, USA) was used to exchange the buffer with PBS and to concentrate the purified antibody. The purified A21G3.3 antibody was biotinlabeled using $10 \times$ mol of EZ-Link Sulfo-NHS-LC Biotin (Pierce Chemical Co., Rockford, Illinois, USA), and incubated at room temperature for 30 minutes. The biotinylation reaction was stopped with $150 \mathrm{mM}$ glycine. The sample was then applied to a desalting column (Amersham Pharmacia Biotech, Uppsala, Sweden) to remove the free biotin.

Flow cytometry. Mice were sacrificed, and spleen and submaxillary glands were collected. Single-cell suspensions were prepared by grinding spleens and lymph nodes between frosted glass slides (Menzel-Glaser GmbH, Braunschweig, Germany). Cells were filtered through a $70-\mu \mathrm{m}$ nylon Falcon cell strainer (Becton Dickinson and Co., Franklin Lakes, New Jersey, USA), and erythrocytes were removed from splenocyte suspensions by osmotic lysis with red blood cell lysis solution $(8.34 \mathrm{mg} / \mathrm{ml}$ ammonium chloride, $0.84 \mathrm{mg} / \mathrm{ml}$ sodium bicarbonate, and $1 \mathrm{mM}$ EDTA, pH 8.0). Submaxillary glands were cut into pieces of $2-3 \mathrm{~mm}$ and incubated in $1 \mathrm{mg} / \mathrm{ml}$ sterile collagenase solution (Roche Diagnostics, Mannheim, Germany) in PBS ( $\mathrm{Ca}^{2+}$ and $\mathrm{Mg}^{2+}$ free) for 1 hour at $37^{\circ} \mathrm{C}$, until leukocytes were released from the tissue. After digestion, the cell suspension was filtered through a $70-\mu \mathrm{m}$ nylon Falcon cell strainer (Becton Dickinson and Co.), and filtered cells were washed twice with PBS. Leukocytes obtained from spleens or submaxillary glands were resuspended in FACS buffer ( $1 \%$ BSA and $0.05 \%$ sodium azide in PBS) at a concentration of $5 \times 10^{6}$ cells $/ \mathrm{ml}$. Surface staining was done using various combinations of antibodies labeled with FITC, phycoerythrin, Cy5, and CyChrome. Fluorescent-labeled anti-mouse antibodies anti-CD4 (L3T4), anti-CD8 $\alpha$ (Ly-2), anti-CD45R/B220 (RA36B2), anti-Ly6-G (GR1), anti-IgD (11-26c.2a), antiCD11b (Mac-1), anti-Ly55 (NK1.1 and NKR-P1C), anti$\operatorname{IgM}(\mathrm{R} 6-60.2)$, anti-CD23 (IgE Fc receptor, clone B3B4), 
anti-CD24 (heat stable antigen, 30F1), anti-CD43 (S7), anti-L-selectin (MEL-14), anti-CD1 (1B1), and antiCD21 (7G6) were supplied by BD Biosciences Pharmingen (San Diego, California, USA). Cy5-conjugated anti-IgM antibody was purchased from Jackson ImmunoResearch Laboratories Inc. (West Grove, Pennsylvania, USA). FITC-labeled antibodies were used at 1:100 dilution; other fluorochrome-labeled antibodies were used at 1:200 final dilution. For flow cytometry, we acquired 30,000-100,000 events per sample. Data was collected on a FACSCalibur flow cytometer, and analyzed using CellQuest software (Becton Dickinson Immunocytometry Systems, San Jose, California, USA). Immunohistochemistry. Submaxillary glands were collected from both control and BAFF Tg mice. Tissues were either frozen in Tissue-Tek OCT compound (Sakura Finetek USA Inc., Torrance, California, USA) or fixed in $10 \%$ buffered formalin and embedded in paraffin. Samples of human labial glands collected by biopsy were fixed and embedded in paraffin blocks by pathologists at the Flinders Medical Centre in Adelaide, Australia. Paraffin sections ( $5 \mu \mathrm{m}$ thick) were rehydrated in successive baths of xylene, $100 \%$ ethanol, and $\mathrm{H}_{2} \mathrm{O}$. Slides were cooked under pressure in citrate buffer $(8.2$ $\mathrm{mM}$ trisodium citrate and $1.7 \mathrm{mM}$ citrate, $\mathrm{pH}$ 6.0). Slides were either stained with hematoxylin and eosin for histologic examination, or used for immunohistochemical staining. Prior to immunohistochemical staining, tissue sections were preincubated with 10 $\mu \mathrm{g} / \mathrm{ml}$ human Ig (Novartis International AG, Basel, Switzerland) in TBS-Triton (0.5\% Triton) to block nonspecific binding, and washed twice with TBS-Triton. Sections were incubated with $5 \mu \mathrm{g} / \mathrm{ml}$ of either rat antihuman BAFF (Buffy-2) or an isotype-matched control rat antibody (BD Biosciences Pharmingen) for $30 \mathrm{~min}-$ utes at room temperature, and washed with TBS-Triton. Slides were then incubated with biotin-labeled rabbit anti-rat Ig (1:100; DAKO Pty. Ltd., Botany, Australia) for 30 minutes at room temperature. This was followed by incubation with horseradish peroxidase-labeled streptavidin (Jackson ImmunoResearch Laboratories Inc.) for 30 minutes, and visualization using the substrate 3,3'-diaminobenzidine (Vector Laboratories Inc., Burlingame, California, USA). Sections were counterstained using hematoxylin (Sigma Chemical Co., St. Louis, Missouri, USA) and Scott's bluing solution, and dehydrated in successive baths of $\mathrm{H}_{2} \mathrm{O}, 100 \%$ ethanol, and xylene. Slides were mounted with coverslips and Eukitt mounting solution (Calibrated Instruments Inc., Hawthorne, New York, USA). Endogenous peroxidase activity was blocked with $2 \%$ hydrogen peroxide in methanol for 20 minutes before staining with the primary antibody. All slides were observed under a Leica light microscope, and images were captured using a Leica DC 200 camera (Leica Microsystems North America, Bannockburn, Illinois, USA).

Scoring of mouse salivary glands for degree of inflammation and tissue destruction. Tissue sections of mouse submaxillary glands stained with hematoxylin and eosin were examined at $\times 100$ under the microscope, and scored as previously described (32). The degree of inflammatory infiltrates was graded as follows: 1 indicated that 1-5 foci of mononuclear cells were seen (more than 20 cells per focus); 2 indicated that more than 5 foci of mononuclear cells were seen, but without significant parenchymal destruction; 3 indicated that multiple confluent foci were seen, with moderate degeneration of parenchymal tissue; and 4 indicated extensive infiltration of the gland with mononuclear cells, and extensive parenchymal destruction.

Measurement of salivary flow. Mice were anesthetized and injected intraperitoneally with $50 \mu \mathrm{g}$ of sterile pilocarpine in PBS (Sigma Chemical Co.) per $100 \mathrm{~g}$ body weight. After 4 minutes, saliva was collected for 5 minutes on a cotton swab. The weight of the cotton swab was measured before and after saliva collection. The amount of saliva collected was normalized to $\mu \mathrm{g}$ saliva per g body weight.

Clinical samples from patients with primary SS, SLE, and $R A$. Sera were obtained from 53 patients with SLE and 53 patients with RA that met the American College of Rheumatology criteria for the classification of disease $(33,34)$. Sera were collected from 41 patients who were followed between 1995 and 2001 at Flinders Medical Centre, and who fulfilled at least four of six European consensus criteria for the diagnosis of primary SS (35). No SS patient had been treated with corticosteroids or immunosuppressive agents. Control sera were collected from 39 healthy donors. Labial salivary gland biopsies, with lymphocyte focus scores of greater than 1 per $4 \mathrm{~mm}^{2}$ of salivary gland tissue (36), were obtained from four patients with primary SS whose sera contained anti-Ro and anti-La autoantibodies. Histologically normal labial salivary gland tissues were obtained from three controls.

ELISA assays for detection of human BAFF in sera from patients with primary SS. Sera from patients were diluted 1:10 in PBS 1\% BSA and precleared of human Ig on protein A-Sepharose beads (10\% vol/vol pelleted beads; Amersham Pharmacia Biotech) overnight at $4^{\circ} \mathrm{C}$. ELISA plates (Nalge Nunc International, Rochester, New York, USA) were coated with $2 \mu \mathrm{g} / \mathrm{ml}$ rat antihuman BAFF antibody (Buffy-5) overnight at $4^{\circ} \mathrm{C}$. After blocking, serial dilutions of the precleared sera were added, followed by the detection antibody, biotinconjugated mouse anti-human BAFF $(0.5 \mu \mathrm{g} / \mathrm{ml}$, clone A21G3.3). Alkaline phosphatase-labeled streptavidin (Jackson ImmunoResearch Laboratories Inc.) and the corresponding alkaline phosphatase substrate Sigma 104 (Sigma Chemical Co.) were used for detection. The reaction was stopped using $3 \mathrm{~N} \mathrm{NaOH}$. Plates were read at an OD of $405 \mathrm{~nm}$, and a standard curve was generated using known quantities of recombinant human BAFF, diluted in human serum and treated as described above for patients' samples.

Statistical analyses were performed using StatView software (Abacus Concept Inc., Cary, North Carolina, USA) and ANOVA. 

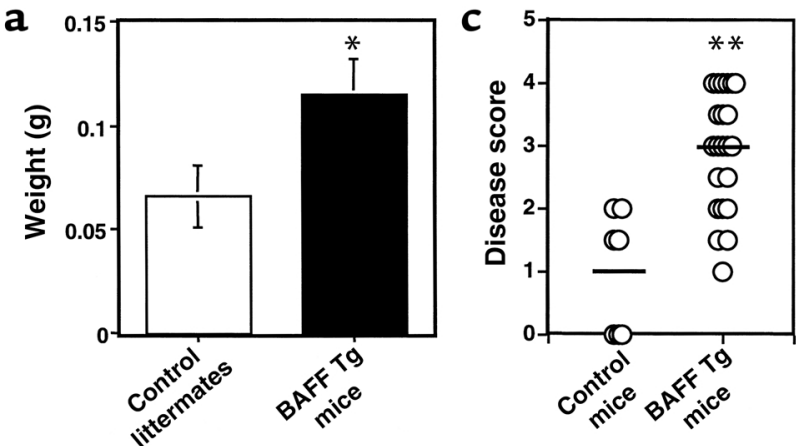

b

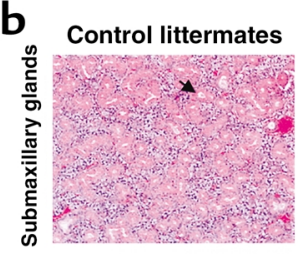

BAFF Tg mice

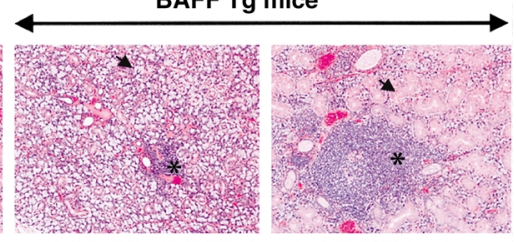

\section{Results}

BAFF Tg mice develop a Sjögren-like syndrome as they age. Following routine dissections of BAFF Tg mice, we observed that many mice over 13 months of age had enlarged salivary (submaxillary) glands (Figure 1a). The cohort of mice over 13 months old was difficult to obtain and maintain, because most BAFF Tg mice had developed signs of severe nephritis by this age. Nevertheless, we were able to analyze more than 50 mice 13-17 months of age, and more than 20 agematched control littermates. Histologically, we observed massive destruction of acinar cells with small periductal foci, as well as large leukocytic infiltrates (Figure 1b, middle). We analyzed histological preparations of 22 BAFF Tg mice, 12-17 months old, and seven control age-matched mice. We scored the extent of the disease using a previously described scoring system (32), which takes into consideration the number of foci of infiltrating leukocytes in the gland and the extent of destruction of the parenchymal tissue. This analysis showed a significantly increased incidence of disease in BAFF Tg mice compared with age-matched controls (Figure 1c). We did not analyze sublingual or parotid salivary glands, but concentrated on the analysis of submaxillary glands. We did not look at lacrimal glands, because mice did not develop obvious ocular disorders (keratoconjunctivitis). Our analysis revealed that $60 \%$ of BAFF Tg mice over 12 months of age showed severe inflammation (grade 3 or above, Figure 1c). It is interesting to note that small foci of infiltrating leukocytes were detected in the submaxillary glands of age-matched control mice, but not in younger control mice (data not shown). However, the disease grades of aged control mice remained at $0-2$. We also saw heterogeneity within the population of BAFF $\mathrm{Tg}$ mice. We found some mice with less destruction of the duct/acinar epithelial cells, but much larger periductal infiltrates (Figure $1 \mathrm{~b}$, right panel). The pathology observed was independent of

\section{Figure 1}

Enlarged and inflamed salivary glands in BAFF Tg mice. (a) Mice 15-17 months old (four control littermates and four BAFF Tg mice) were sacrificed the same day for organ collection. Both right and left submaxillary glands were collected and weighed. The data shows the combined weight (mean \pm SD) of both glands. These results are representative of at least four separate groups of dissected age-matched animals. (b) Paraffin sections of submaxillary glands from a control littermate (left panel) and two BAFF Tg mice (middle and right panels) were stained with hematoxylin and eosin. Arrows indicate ducts and acinar cells in the left and right panels. The arrow in the middle panel shows acinar destruction. Asterisks indicate periductal infiltrates (foci). Magnification: $\times 100$. (c) Paraffin sections of submaxillary glands from seven control mice and 22 BAFF Tg mice (12-17 months old) were prepared as shown in $\mathbf{b}$ and scored for disease as described in Methods. Bars indicate the mean disease score for each group. ${ }^{*} P<0.05,{ }^{*} P<0.03$.

the sex of the animals (data not shown). Interestingly, we observed three cases in which a BAFF Tg mouse, 13-15 months of age, had a large submaxillary tumor (more than $1 \mathrm{~cm}$ in diameter). Histological analysis revealed that these tumors contained hyperplastic lymphoid tissue composed mainly of activated B lymphocytes and numerous germinal centers (data not shown). These abnormal aggregates of B lymphoid cells were also seen in the lymphocytic infiltrates found in the salivary glands of another four BAFF Tg mice (data not shown). Whether these cells are malignant or not is unknown, and the very low frequency of tumors has not yet permitted more extensive analysis of these cells.

Submaxillary glands from older BAFF Tg mice are infiltrated by a large number of $B$ cells. We selected seven BAFF Tg mice that had significantly larger submaxillary glands than control mice had. One of the two glands collected from each mouse was digested with collagenase, and mononuclear cells from these glands were purified and analyzed by flow cytometry. Absolute counts of various cell types showed a consistent and significant increase in the number of $B$ cells infiltrating the submaxillary glands of aging BAFF Tg mice (Table 1). Numbers of T cells, NK cells, macrophages, and granulocytes also increased; however, there was much greater variation between animals (Table 1) than in the control group. Hematoxylin and eosin staining done on the second gland collected from each $\mathrm{Tg}$ animal confirmed that the disease score on these tissues was at least 3 for each BAFF Tg mouse selected (data not shown). We tested the presence of anti-Ro and anti-La autoantibodies in sera of BAFF Tg mice that had severe lesions in their submaxillary glands, in a specific ELISA assay using recombinant full-length mouse Ro52, mouse Ro60, and mouse La antigens (37). These mice (with disease grade above 3) did not secrete anti-Ro/SSA and/or anti$\mathrm{La} / \mathrm{SSB}$ autoantibodies, which are often associated with human SS (data not shown). 
A large proportion of $B$ cells infiltrating submaxillary glands of BAFF Tg mice have an MZ-like phenotype. We did a profiling analysis by flow cytometry of the $\mathrm{B}$ cells infiltrating the submaxillary glands of BAFF Tg mice, and analyzed two $B$ cell subsets based on their level of expression of B220 (Figure 2a). Analysis of the B-1 $\mathrm{B}$ cell population in the $\mathrm{B} 220 \mathrm{low} /$ intermediate (B220 $0^{\mathrm{lo} / \text { int }}$ gate revealed a threefold increase of $\mathrm{B}-1 \mathrm{~b}$ $\left(\mathrm{CD5}^{-}\right) \mathrm{B}$ cells, and the presence of $\mathrm{B}-1 \mathrm{a}\left(\mathrm{CD}^{+}\right) \mathrm{B}$ cells that were not detected in control mice (Figure $2 \mathrm{~b}$ ). In the $\mathrm{B} 220$ high $\left(\mathrm{B} 220^{\mathrm{hi}}\right)$ gate, cells were analyzed according to their level of IgM expression. The IgM dull $\left(\mathrm{IgM}^{\text {dull }}\right)$ subset of $B$ cells is present in both control and BAFF Tg mice, but is larger in BAFF Tg mice than in control mice (Figure $2 \mathrm{~b}$ ). The IgMdull subset in both control and BAFF Tg mice contains B-2-like cells, which are $\mathrm{CD} 21^{\mathrm{lo} / \mathrm{int}}, \mathrm{IgD}^{+}$, and $\mathrm{CD}_{2} 3^{+}$(data not shown). The $\mathrm{B} 220^{\mathrm{hi}} / \mathrm{IgM}^{\mathrm{hi}} \mathrm{B}$ cell subset was greatly increased in BAFF Tg mice compared with control mice (Figure $2 \mathrm{~b}$ ). Moreover, the IgM $\mathrm{Ig}^{\text {hi }} \mathrm{B}$ cells found in BAFF $\mathrm{Tg}$ mice are phenotypically different than IgM ${ }^{\text {hi }} \mathrm{B}$ cells from control mice. Indeed, the small $\operatorname{IgM}^{\text {hi }} \mathrm{B}$ cell subset detected in control mice had characteristics of B-2 $\mathrm{B}$ cells $\left(\mathrm{CD} 21^{\mathrm{lo} / \text { int }}, \mathrm{IgD}^{+}\right.$, and $\mathrm{CD}^{2} 3^{+}$; Figure $\left.2 \mathrm{e}\right)$, whereas the large IgM ${ }^{\text {hi }} \mathrm{B}$ cell subset of BAFF Tg mice had no counterpart in control mice, and resembled MZ B cells in many respects. These cells were $\mathrm{IgD}^{\mathrm{lo}}, \mathrm{CD} 21^{\mathrm{hi}}$, $\mathrm{CD} 23^{\mathrm{lo} /-}, \mathrm{CD} 1^{\mathrm{hi}}, \mathrm{HSA}^{++}$(Figure 2e), and L-selectin ${ }^{\text {lo }}$ (Figure 2d). These cells also express lower levels of CD43 than do B-1 B cells (Figure 2c). Because their profile was closer to that of MZ B cells than to other phenotypically related B cell subsets, such as B-1 and splenic transitional T1 B cells, we refer to these cells as MZ-like B cells (Figure 2f) $(38,39)$. To summarize, we have identified a new population of $B$ cells, which most closely resemble MZ B cells, infiltrating the salivary glands of BAFF $\mathrm{Tg}$ mice. These cells are $\mathrm{IgM}^{\mathrm{hi}}, \mathrm{B} 220^{\mathrm{hi}}$, $\mathrm{CD} 5^{-}, \mathrm{CD} 23^{\mathrm{lo} /}, \mathrm{IgD}^{\mathrm{lo} /}, \mathrm{CD} 1^{\mathrm{hi}}, \mathrm{CD} 21^{\mathrm{hi}}$, $\mathrm{L}_{\text {-selectin }}{ }^{\mathrm{lo}}$, and $\mathrm{HSA}^{++}$. However, these cells also have some slight similarities with B-1b cells, such as low expression of CD43, although less than on B-1 cells.

Older BAFF Tg mice have reduced saliva production. To determine whether the alterations seen in tissue sections of submaxillary glands from BAFF $\mathrm{Tg}$ mice have any effect on saliva flow, we injected $13 \mathrm{BAFF}$ Tg mice and 14 control littermates, aged $8-15.5$ months, with pilocarpine 4 minutes prior to the measurement of saliva flow. In general, the saliva flow in older control mice (aged 13-15.5 months) was higher than in control mice aged $8-10$ months (Figure 3 , $a$ and $b$, respectively). The reason for this is unclear, but it may be related to the weight of the mice, which increases with age. Since saliva production is age-dependent in this assay, it is important to compare control mice and BAFF Tg mice within the same age group. Therefore, we divided mice into two age groups, $8-10$ months (Figure $3 \mathrm{~b}$ ) and 13-15.5 months (Figure 3a). We found that mice 13-15.5 months of age produced significantly less saliva than did age-matched control animals (Figure 3a). However, this difference was not seen with mice 8-10 months of age (Figure $3 b$ ). Reduction in saliva flow correlated with the higher number of $\mathrm{B}$ lymphocytes detected in submaxillary glands of these animals (data not shown).

Elevated levels of human BAFF detected in the serum of patients suffering from primary SS. Because the Sjögrenlike pathology observed in some BAFF Tg mice had features similar to those found in human SS, we measured BAFF levels in sera of patients suffering from this disease, and compared them with levels in patients suffering from SLE and RA. Sera from 53 patients with SLE,

Table 1

Analysis of leukocytes present in salivary glands of BAFF Tg mice and control littermates by flow cytometry

\begin{tabular}{|c|c|c|c|c|c|c|c|}
\hline $\begin{array}{c}\text { Age } \\
\text { (months) }\end{array}$ & $\begin{array}{l}\text { Total leukocytes } \\
\qquad\left(\times 10^{6}\right)\end{array}$ & $\begin{array}{l}\text { B cells } \\
\left(\times 10^{6}\right)\end{array}$ & $\begin{array}{l}\mathrm{CD}^{+}{ }^{+} \mathrm{T} \text { cells } \\
\left(\times 10^{6}\right)\end{array}$ & $\begin{array}{l}\mathrm{CD}^{+} \mathrm{T} \text { cells } \\
\left(\times 10^{6}\right)\end{array}$ & $\begin{array}{l}\text { NK cells } \\
\left(\times 10^{6}\right)\end{array}$ & $\begin{array}{l}\text { Mac- } 1^{+} \text {cells } \\
\quad\left(\times 10^{6}\right)\end{array}$ & $\begin{array}{l}\mathrm{GR}^{+}{ }^{+} \text {cells } \\
\left(\times 10^{6}\right)\end{array}$ \\
\hline
\end{tabular}

Control

$\left(\times 10^{6}\right)$

$\left(\times 10^{6}\right)$

$\left(\times 10^{6}\right)$

littermates

Control 1
Control 2
Control 3
Control 4

Mean \pm SD

BAFF Tg mice

BAFF Tg 1

BAFF Tg 2

BAFF Tg 3

BAFF Tg 4

BAFF Tg 5

BAFF Tg 6

BAFF Tg 7

Mean \pm SD

$P$ value

14
14
14
15

$14.25 \pm 0.5$

14
14
16
8
16
17
16
$14.4 \pm 3$

$\begin{array}{cc}8.4 & 0.048 \\ 5.6 & 0.045 \\ 5.9 & 0.028 \\ 1.5 & 0.0014 \\ 5.35 \pm 2.8 & 0.03 \pm 0.02\end{array}$

$$
\begin{gathered}
30 \\
31.7 \\
42.3 \\
6 \\
11 \\
2.5 \\
22 \\
21 \pm 14 \\
P<0.05
\end{gathered}
$$

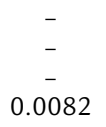

1.1

0.14

0.6

0.35

4.6

0.03

9.3

$2.3 \pm 3.5$

$P<0.05$

$\begin{array}{cccc}\text { ND } & 0.04 & 0.22 & \text { ND } \\ \text { ND } & 0.03 & 0.17 & \text { ND } \\ \text { ND } & 0.02 & 0.23 & \text { ND } \\ 0.0011 & 0.02 & 0.57 & 0.062 \\ 0.027 \pm 0.009 & 0.29 \pm 0.18 & & \\ & & & \\ \text { ND } & 0.27 & 0.7 & \text { ND } \\ \text { ND } & 0.31 & 1.03 & \text { ND } \\ \text { ND } & 0.02 & 0.23 & \text { ND } \\ 0.028 & 0.13 & 0.29 & 0.45 \\ 1.6 & 0.64 & 0.27 & 4.3 \\ 0.022 & 0.087 & 0.17 & 0.23 \\ 1.6 & 1.4 & 0.5 & 8.7 \\ & 0.41 \pm 0.5 & 0.46 \pm 0.31 & \\ & & & \end{array}$

Animals were sacrificed and submaxillary glands were dissected. Tissues were digested and leukocytes were purified as described in Methods. The total number of leukocytes per gland was counted using a hemocytometer. Cells were stained with anti-B220 (B cells), anti-CD4 and anti-CD8 (T cells), anti-NK1.1 (NK cells), anti-Mac-1 (macrophages), and anti-GR1 (granulocytes) fluorochrome-labeled antibodies. Flow cytometry analysis provided percentage values for each subpopulation of cells, and absolute numbers were calculated relative to the total number of cells collected per gland. ND, not detected. -, no analysis done. Only significant $P$ values are indicated. 
a

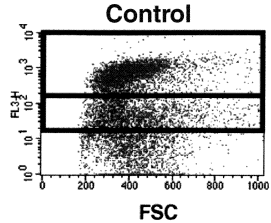

b

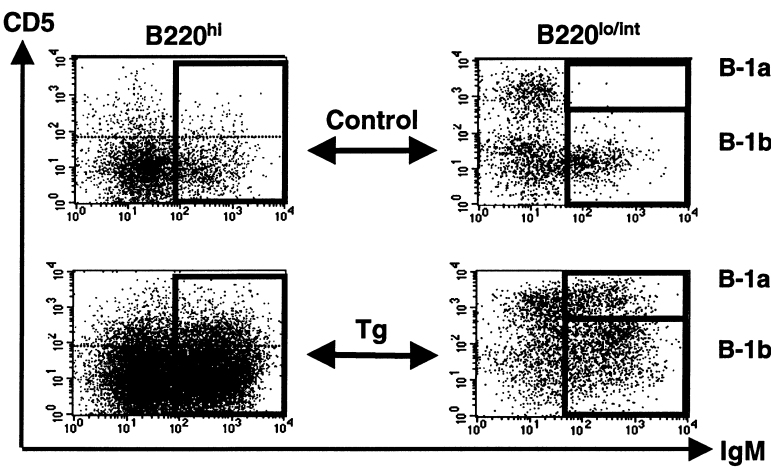

c

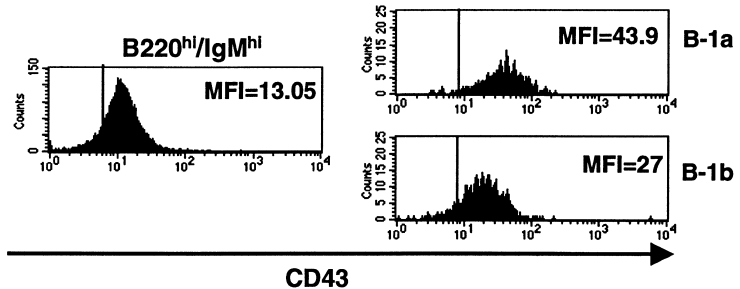

d

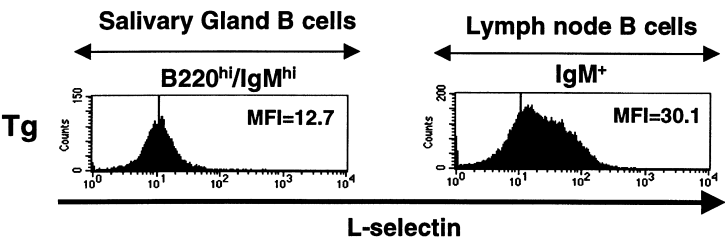

e
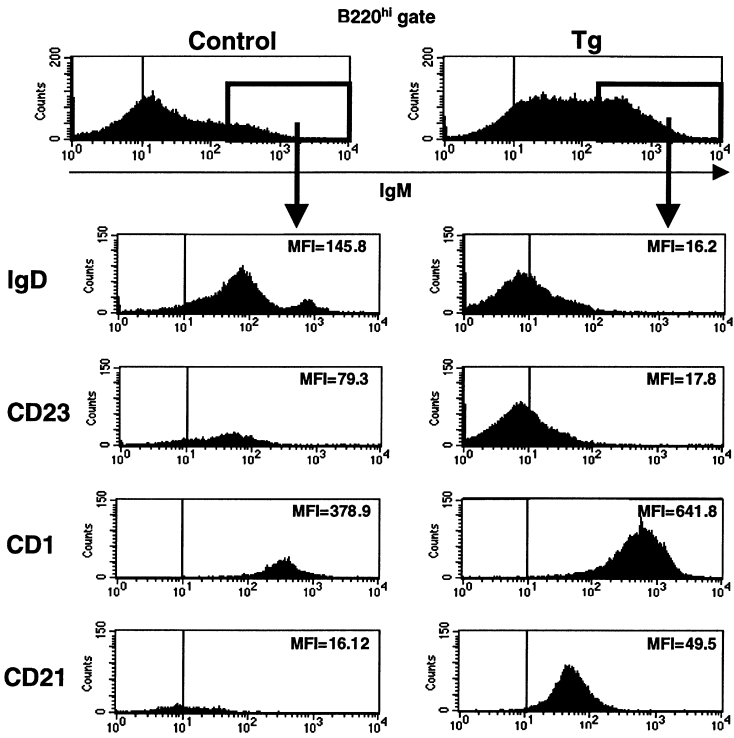

HSA
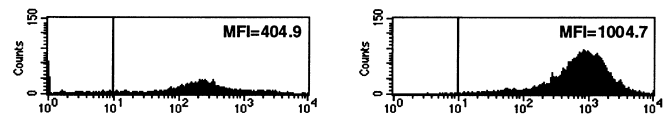

f

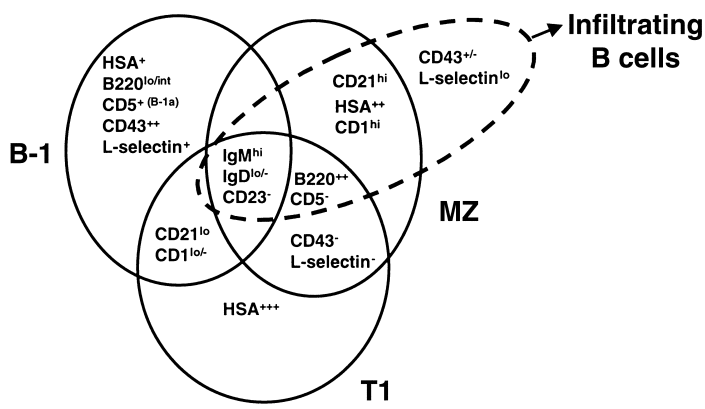

Figure 2

Identification of MZ-like B cells infiltrating salivary glands of BAFFTg mice. Lymphocytes were isolated from salivary glands of BAFF Tg mice (13-17 months old) and control littermates. Cells were stained with anti-B220, anti-CD5, anti-IgM, and anti-CD43 using multi-color flow cytometry. (a) Gates used to identify B220 hi and B220 lo/int B cells in control and BAFF Tg mice. (b) Expression of IgM and CD5 on B220 hi cells and B220 $0^{\mathrm{l} / \text { int }}$ cells from BAFF Tg and control mice. The IgM $\mathrm{M}^{\text {hi }} \mathrm{B}$ cell gate on B220 $0^{\text {hi }}$ cells is boxed and the B-1a and B-1b gates on $\mathrm{B} 220^{\mathrm{lo} / \text { int }}$ cells are indicated. (c) Expression of CD43 on gated B220hi/lgMhi cells and gated B-1a and B-1b cells from a BAFF Tg mouse. (d) L-selectin expression on lymphocytes from salivary gland and inguinal lymph node of a BAFF Tg mouse, gated on B220 hi/lgM hi cells and on $\mathrm{IgM}^{+}$cells. (e) Lymphocytes from a BAFF Tg mouse and a control littermate were prepared as in a and stained with anti-B220, anti-IgM, and antibodies to the indicated markers. Cells were gated on B220 hi and IgM $\mathrm{M}^{\text {hi }}$ cells, as shown in the top histograms. In c-e, mean fluorescence intensity (MFI) and bars delineating negative control staining are indicated. (f) Schematic representation of the common and distinct markers expressed on MZ B cells (right circle), B-1 cells (left circle), and T1 B cells (lower circle). The phenotype of the B cells infiltrating submaxillary glands of BAFF Tg mice is represented by the dotted ellipse. These results are representative of 12 BAFF Tg mice and seven control mice analyzed. FSC, forward light scatter.

53 patients with RA, 41 patients with primary SS, and 39 healthy control individuals were analyzed using a human BAFF-specific ELISA assay. This assay showed that at least 15 of 41 patients with SS $(36 \%)$ clearly had higher levels of serum BAFF than were found in healthy control individuals (Figure 4a). The percentages of RA and SLE patients with elevated BAFF levels in their blood were $19 \%$ and $23 \%$, respectively. The mean BAFF level was higher in patients with SS than in patients with SLE or RA (Figure 4a). Two patients with SS had very high BAFF levels (over $200 \mathrm{ng} / \mathrm{ml}$, Figure 4a). BAFF levels in patient sera did not correlate with the levels of total IgG or rheumatoid factors (Figure 4, b and c, respectively). Additionally, these BAFF levels did not correlate with the presence of precipitins such as anti-Ro or anti-La (Figure 4d). We confirmed human BAFF levels in patient sera using BAFF-specific immunoprecipitation procedures, followed by Western blotting techniques (data not shown).

Detection of BAFF-expressing cells in tissue sections from labial glands of patients with SS. We checked BAFF expression at the site of abnormal lymphocyte infiltrates in biopsies of labial glands from SS patients. A strong expression of BAFF was detected on leukocytic infiltrates, and 
on some stromal elements such as ductal epithelial cells, although clearly not all cells within the infiltrate were positive for BAFF (Figure 4e, right panel). The antibody did not stain normal labial salivary gland tissue (Figure $4 \mathrm{e}$, bottom left), and addition of recombinant human BAFF competed for the staining (data not shown). We also stained tissue sections of labial salivary glands from patients with sarcoidosis and chronic sialadenitis. BAFF staining was far less intense in tissues from patients with sarcoidosis than in tissues of SS patients; staining was mainly present on some mononuclear cells disseminated in the fibrotic tissues, and appeared weakly on some stromal elements (data not shown). We did not detect BAFF expression in tissues from patients with chronic sialadenitis (data not shown).

\section{Discussion}

The B cell disorder seen in patients with SS is particularly severe compared to B cell disfunction seen in other autoimmune diseases such as SLE and RA. Moreover, patients with SS are especially prone to the development of B cell lymphomas (1), which suggests that dysregulation of $\mathrm{B}$ cell tolerance, survival, and differentiation may be a central defect in this disease.

We show that BAFF Tg mice and patients with SS share several important clinical features. Inflammation of the salivary glands and the associated exocrinopathy in BAFF Tg mice appeared late in life, and secondary to an SLE-like pathology. Human SS can also develop secondary to SLE in some patients as they age (1). Similar to patients with SS, BAFF Tg mice secreted large amounts of rheumatoid factors and Ig's $(23,40)$. B cells from these mice were hyperreactive, and present in large numbers in the blood and secondary lymphoid organs (23). BAFF Tg mice also developed splenomegaly and adenopathy (23), and had enlarged salivary glands (submaxillary glands). In older BAFF Tg mice, secretion of saliva was significantly reduced compared with that of age-matched control mice. The histological analysis of submaxillary glands from BAFF $\mathrm{Tg}$ mice revealed the presence of numerous focal lymphocytic infiltrates, associated with varying degrees of acinar destruction. Therefore, according to the guidelines for the diagnosis of SS based on the European criteria (35), BAFF Tg mice developed a pathology similar to human SS. Interestingly, B-lymphoid hyperplasia was detected in the salivary glands and submaxillary tumors of some BAFF Tg mice. This observation is intriguing, as Sjögren's disease in humans is associated with a higher risk of developing lymphomas (1). More studies are required to determine the exact nature of the hyperplastic B-lymphoid clusters found in the salivary glands of some BAFF Tg mice. Lymphocytic infiltrates were also detected in the lungs and liver of some aging BAFF Tg mice (data not shown). Nevertheless, some important features differentiate the pathology seen in BAFF Tg mice and human SS. Many patients with SS secrete the characteristic autoantibodies directed against ribonucleoprotein particles, anti-Ro (SS-A) and anti-La (SS-B) (1). These autoantibodies were not detected in BAFF $\mathrm{Tg}$ mice with severe sialadenitis. In human SS and some animal models, $\mathrm{CD} 4^{+} \mathrm{T}$ cells are usually the predominant population infiltrating salivary glands, although significantly higher numbers of B cells are also present in inflamed glandular tissues (2). Moreover, the presence of B cells seems to play a major role in the exocrinopathy seen in several animal models of SS (41). In contrast, an overwhelming number of B cells compared with $T$ cells infiltrated submaxillary glands of BAFF Tg mice. To summarize, aging BAFF Tg mice display a disease with all the qualifying features of SS with additional characteristics seen in humans, which distinguish this model from previously described mouse models of SS.

The etiology of SS, both in BAFF Tg mice and in humans, is still unclear, but may relate directly to the effect of BAFF on B cell differentiation. We demonstrated previously that BAFF specifically supports cell survival of splenic immature T2 B cells (20). We also hypothesized that maturation of $\mathrm{B}$ cells in the spleen is a critical immune checkpoint, providing censoring death signals to emerging autoreactive immature B cells (20). In BAFF Tg mice, we believe that excess survival signals provided by BAFF, via upregulation of Bcl-2 (23), eliminated the censoring death signals. In support of this notion, mice that overexpress $\mathrm{Bcl}-2$ in $\mathrm{B}$ cells, or that are deficient in a Bcl-2-related mediator of apoptosis known as Bim, develop an autoimmune condition very similar to the SLE-like disease seen in BAFF $\mathrm{Tg}$ mice $(42,43)$. Moreover, lymphocytic infiltrates and Sjögren-like disorders have been detected in the salivary glands of Bcl-2 Tg mice (42). Therefore, excess B cell survival is a key event in SLE- and Sjögren-related disorders.

Excessive survival signals led to the massive expansion of the T2 B cell subset, but also the MZ B cell subset in BAFF Tg mice (20), supporting the possibility

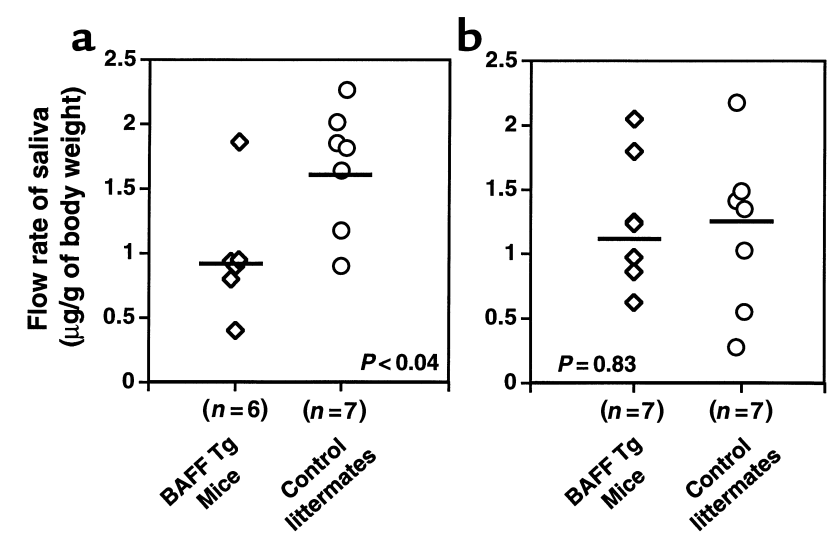

Figure 3

Decreased saliva flow in older BAFF Tg mice. Thirteen BAFF Tg mice (diamonds) and 14 control littermates (circles) were injected with pilocarpine prior to saliva collection as described in Methods. (a) Mice 13-15.5 months old. (b) Mice 8-10 months old. Mean values for saliva flow are shown with a bar. $P$ values are indicated in each panel. 

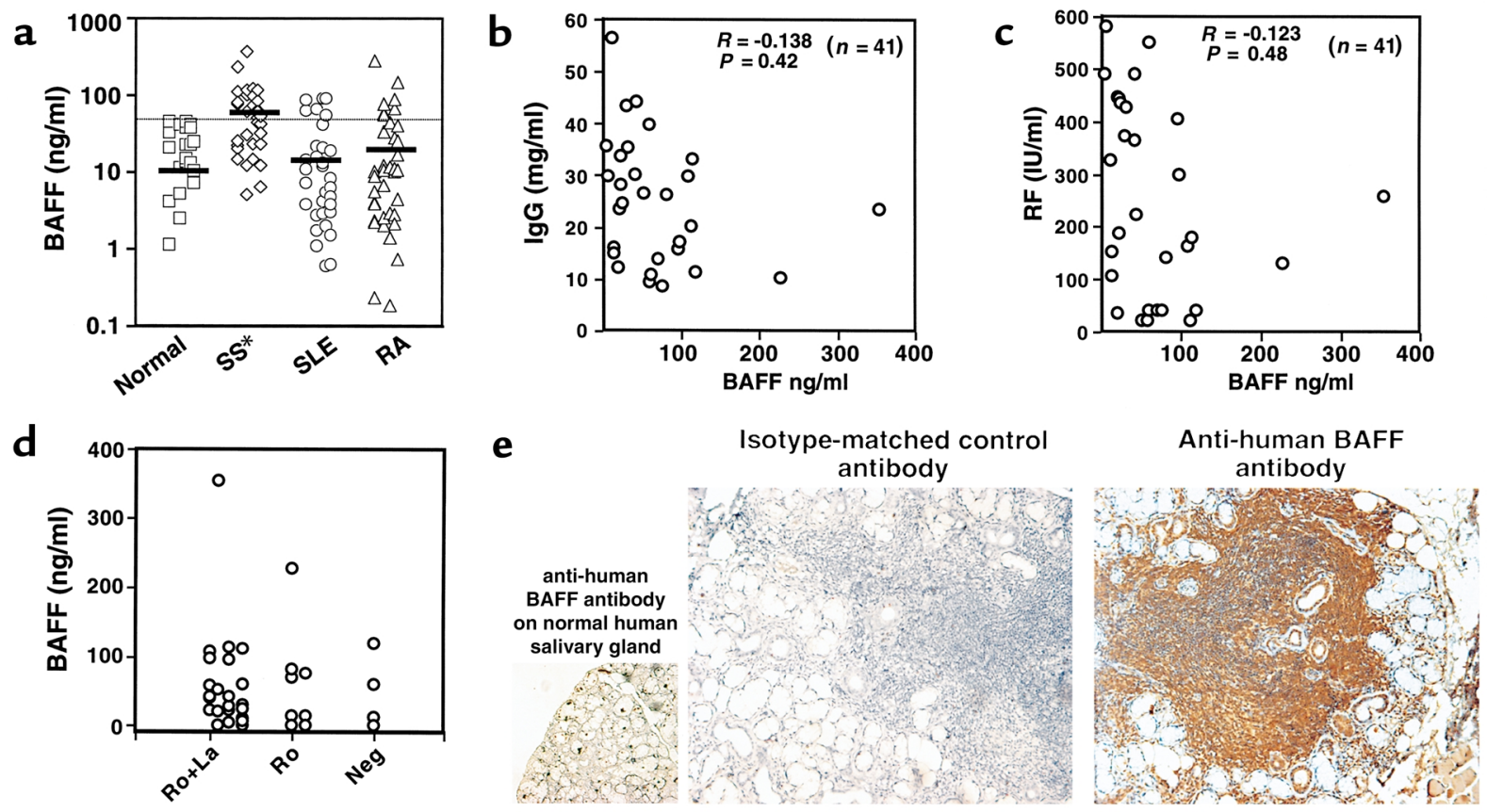

\begin{abstract}
Figure 4
Elevated levels of BAFF in sera and salivary gland tissues from patients suffering from primary SS; no correlation with levels of total IgG, RF, and presence of anti-Ro/La autoantibodies. (a) Individual serum BAFF levels in 39 healthy controls (squares), 41 patients with primary SS (diamonds), 53 patients with SLE (circles), and 53 patients with RA (triangles) were measured by ELISA. The horizontal black bars indicate the mean for each group: normal $10.4 \pm 13(\mathrm{ng} / \mathrm{ml})$; SS $53 \pm 67(\mathrm{ng} / \mathrm{ml})$; SLE $12.7 \pm 24.4(\mathrm{ng} / \mathrm{ml})$; and RA $23 \pm 47(\mathrm{ng} / \mathrm{ml})$. Sera of $16 \mathrm{nor}-$ mal individuals, $6 \mathrm{SS}, 20 \mathrm{SLE}$, and $10 \mathrm{RA}$ patients had no detectable levels of BAFF. The dotted line delineates the range of normal BAFF levels. ${ }^{*} P<0.04$, as determined by ANOVA. (b and $\left.\mathbf{c}\right)$ Correlation of serum BAFF levels in patients with SS with the levels of IgG (b) and RF (c) in each serum sample. $R$ and $P$ values were calculated by ANOVA. (d) Levels of BAFF in patients with anti-Ro plus anti-La or anti-Ro only, and in patients with no precipitin detected (Neg). (e) Paraffin sections of a human labial gland biopsy from a patient with SS were stained with anti-human BAFF antibody or an isotype-matched control antibody. Magnification: $\times 200$. Staining of normal human labial gland with anti-human BAFF antibody is also shown (magnification: $\times 100$ ). These pictures are representative of four patients with primary SS and three control tissues analyzed. RF, rheumatoid factors.
\end{abstract}

that in some circumstances, T2 B cells may differentiate directly into MZ B cells in the spleen $(24,25)$. This work also demonstrated that $\mathrm{T} 2$ and $\mathrm{MZ} \mathrm{B}$ cells are $\mathrm{CD} 1^{\text {hi }}$, a subpopulation shown to contain autoreactive $B$ cells in a mouse model of $\operatorname{SLE}(20,39,44)$. These findings strongly suggested that $\mathrm{T} 2$ and/or MZ B cells might play a central role in the progression of autoimmunity in BAFF Tg mice. Recent work has shown that treatment of mice with $17 \beta$-estradiol (E2) induces a lupus-like condition in $\mathrm{BALB} / \mathrm{c}$ mice that is associated with the expansion of the MZ B cell compartment (45). This work emphasized the potential role of MZ B cells in the development of autoimmune disorders.

We identified a population of $B$ cells infiltrating the salivary glands of BAFF Tg mice that had a markedly different phenotype from the few $B$ cells purified from glands of control animals. $\mathrm{B}$ cells detected in control tissues resembled conventional B-2 B cells. In contrast, salivary glands from BAFF $\mathrm{Tg}$ mice contained a subpopulation of B cells with an MZ-like phenotype. Evidence for an MZ-like phenotype rather than a B-1-like phenotype is provided by the higher expression of CD21, CD1, B220, and HSA on these cells, which was also reported previously for splenic MZ B cells (20, 38,
39). It is conceivable that this population is derived from the expanded MZ B cell population present in the spleen of BAFF $\mathrm{Tg}$ mice. In support of this notion, we have shown previously that MZ B cells were abnormally present in the blood and lymph nodes of BAFF Tg mice (20). This observation suggested that these cells had aberrantly acquired homing receptors, allowing them to circulate and home to lymph nodes. Therefore, it is possible that MZ-like B cells found in the salivary glands of BAFF $\mathrm{Tg}$ mice are derived from abnormally emigrating splenic CD1 $1^{\text {hi }} \mathrm{MZ}$ B cells. These unusual MZ-like $B$ cells, although sharing many of the features displayed by splenic MZ B cells (Figure $2 \mathrm{f}$ ), expressed low levels of CD43, a marker usually expressed on B-1 cells at higher levels (38). Therefore, we cannot completely exclude the possibility that MZ-like B cells are derived from the B-1 B cells also found in slightly higher numbers in the $\mathrm{B} 220^{\mathrm{lo} / \text { int }} \mathrm{B}$ cell population that has been purified from salivary glands of BAFF $\mathrm{Tg}$ mice.

There is increasing evidence of a role for B-1 B cells in autoimmunity (46). In addition, the lymphomalignancy seen in human SS seems to be linked to hyperstimulation of B-1 cells (47). However, analysis in BAFF-deficient mice $(21,22)$ and in several models of 
BAFF Tg mice (48) has failed to show any critical effect of BAFF on the B-1 B cell compartment. This indicates that the new $B$ cell subset described here might be a separate population from B-1 B cells. In one model of BAFF Tg mice that used a lymphoid-specific promoter, the B-1 B cell compartment was enlarged (29). However, recent work has shown that a factor other than BAFF interacting with TACI might be responsible for B-1 cell development/survival (22). These last two points indicate that a fine line exists between $M Z$ and B-1 B cells, especially in the context of the BAFF system. Increased numbers of $M Z B$ cells have been reported in other murine models, but no clear correlation between that event and tissue damage has been made $(45,48)$. In this report, we showed that B cells with a phenotype similar to that of MZ B cells massively infiltrated salivary glands of BAFF Tg mice, and may have contributed to tissue damage. In addition, high levels of BAFF present in the blood of patients with autoimmune disorders may have altered the biology of a similar splenic B cell subset, which further developed into pathogenic $B$ cells. It will, therefore, be very interesting to investigate $M Z B$ cells in more detail and to determine their potential as a source of pathogenic $B$ cells in autoimmune diseases. Moreover, identification of a human counterpart of MZ-like B cells may be useful for the development of cell-specific treatments for autoimmune disorders.

The relevance of BAFF secretion for human SS was suggested by the high levels of BAFF in the serum of many patients with primary SS. These findings were interesting because they revealed some critical differences from other B cell-dependent rheumatic diseases. Previous studies have shown that about $20-25 \%$ of patients with SLE or RA have elevated levels of BAFF in their blood $(26,27)$. We tested sera of RA and SLE patients, and confirmed these findings in terms of BAFF levels (Figure 4a). In contrast, we saw both a higher incidence (36\%) and usually higher levels of BAFF in the serum of patients with SS. These findings are very interesting, as they fit the general observation that $B$ cell function is more severely affected in patients with SS than in patients with SLE or RA (1). For instance, serum Ig levels and the risk of B cell lymphomas are usually highest in patients with SS (1). These data suggest that SS may be a disease more dominantly linked to abnormal BAFF secretion than to RA or SLE. Development of SS is strongly associated with MHC class II genes, in particular the HLA-DR and -DQ alleles (1). The MHC-mediated risk appears to correlate with production of anti-Ro and anti-La autoantibodies (49). Since the presence of high BAFF levels in the blood of patients with primary SS does not correlate with production of these autoantibodies, we think that these two events might be independent. However, the HLA linkage leading to activation of specific $\mathrm{T}$ cells and excessive BAFF stimulation of B cells, and/or the emergence of autoreactive $B$ cells, may be two separate events that when combined may contribute to the development of SS. The factors responsible for overproduction of BAFF are unknown, but they might be related to genetic polymorphisms in the BAFF gene. Interestingly, high expression of BAFF was found in lymphocytic infiltrates in salivary gland tissue sections from patients with SS. Similarly, high levels of BAFF were detected in the synovial fluid of patients with RA, and these exceeded levels found in the blood (27).

Autoimmune diseases such as RA have often been associated with chronic infection. The connection between infection and autoimmune disorders has always been unclear. It is conceivable that inflammation/infection via production of inflammatory cytokines, such as IFN- $\gamma(6)$, triggers BAFF secretion in affected tissues, which in turn stimulates B cell and possibly $\mathrm{T}$ cell responses locally as well as in neighboring lymph nodes. In chronic inflammation, BAFF levels may gradually increase in the blood to levels that affect $B$ cell maturation and immune tolerance in the spleen. This may contribute in some individuals to the development of autoimmune disorders. Conversely, in mice, expression of the BAFF transgene artificially raised levels of BAFF in all tissues, which may have contributed to triggering a pathological condition similar to that seen in humans. Unfortunately, biopsy samples taken from SS patients, primarily fixed and processed for histology, are not available for cell preparation and profiling of infiltrating lymphocytes. However, it is important to note that the most common malignancy associated with SS is an MZ B-cell lymphoma that occasionally arises in the parotid gland of some patients (50). Whether MZ-like B cells also play a role in human SS is still unknown; a better characterization of B cell subsets in humans may provide some important clues.

This study provides an interesting parallel between an SS-like pathology that emerges in BAFF Tg mice and high levels of BAFF found in a large proportion of patients with primary SS. These results support the idea that an imbalance in BAFF production could be a major factor contributing to the development of SS, through the recruitment of a specific and potentially pathogenic subpopulation of $B$ cells that targets exocrine glands. Development of BAFF antagonist reagents, and/or neutralization of these newly described B cells, may offer a new avenue for the treatment of this disease.

\section{Acknowledgments}

We would like to thank Jeffrey Browning, Jennifer Gommerman, Laila Girgis, and Edith Gardiner for critical reading of the manuscript. We also thank Jenny Turner for pathological assessment of our specimens. This work was supported by the Glazebrook Trust and Biogen Inc.

\footnotetext{
1. Jonsson, R., Haga, H.-J., and Gordon, T.P. 2000. Sjögren's syndrome. In Arthritis and allied conditions. W. Koopman, editor. Lippincott Williams \& Wilkins. Philadelphia, Pennsylvania, USA. 1736-1759.

2. Manoussakis, M.N., Talal, N., and Moutsopoulos, H.M. 1998. Sjögren's syndrome. In The autoimmune diseases. N.R. Rose and I.R. Mackay, editors. Academic Press. San Diego, California, USA. 381-404.
} 
3. MacSween, R.N.M., et al. 1967. Occurrence of antibody to salivary duct epithelium in Sjögren's disease, rheumatoid arthritis, and other arthritides. A clinical and laboratory study. Ann. Rheum. Dis. 26:402-411.

4. Haneji, N., et al. 1997. Identification of $\alpha$-fodrin as a candidate autoantigen in primary Sjögren's syndrome. Science. 276:604-607.

5. Stott, D.I., Hiepe, F., Hummel, M., Steinhauser, G., and Berek, C. 1998 Antigen-driven clonal proliferation of B cells within the target tissue of an autoimmune disease. The salivary glands of patients with Sjögren's syndrome. J. Clin. Invest. 102:938-946.

6. Nardelli, B., et al. 2000. Synthesis and release of B-lymphocyte stimulator from myeloid cells. Blood. 97:198-204.

7. Ware, C. 2000. APRIL and BAFF connect autoimmunity and cancer. J. Exp. Med. 192:F35-F37.

8. Ware, C.F. 2000. Decoy receptors thwart B cells. Nature. 404:949-950.

9. Laabi, Y., and Strasser, A. 2000. Lymphocyte survival-ignorance is BLyS Science. 289:883-884.

10. Khare, S.D., and Hsu, H. 2001. The role of TALL-1 and APRIL in immune regulation. Trends Immunol. 22:61-63.

11. Yu, G., et al. 2000. APRIL and TALL-1 and receptors BCMA and TACI: system for regulating humoral immunity. Nat. Immunol. 1:252-256.

12. Wu, Y., et al. 2000. Tumor necrosis factor (TNF) receptor superfamily member TACI is a high affinity receptor for TNF family members APRIL and BLyS. J. Biol. Chem. 275:35478-35485.

13. Shu, H.-B., and Johnson, H. 2000. B cell maturation protein is a receptor for the tumor necrosis factor family member TALL-1. Proc. Natl. Acad. Sci. USA. 97:9156-9161.

14. Thompson, J.S., et al. 2001. BAFF-R, a novel TNF receptor that specifically interacts with BAFF. Science. 293:2108-2111.

15. Von Buelow, G.U., and Bram, R.J. 1997. NF-AT activation induced by a CAML-interacting member of the tumor necrosis factor receptor superfamily. Science. 278:138-141.

16. Xia, X.-Z., et al. 2000. TACI is a TRAF-interacting receptor for TALL-1, a tumor necrosis factor family member involved in B cell regulation. J. Exp. Med. 192:137-143.

17. Yan, M., et al. 2000. Identification of a receptor for BLyS demonstrates a crucial role in humoral immunity. Nat. Immunol. 1:37-41.

18. Thompson, J.S., et al. 2000. BAFF binds to the TNF receptor-like molecule BCMA and is important for maintaining the peripheral B cell population. J. Exp. Med. 192:129-135.

19. Do, R.K.G., et al. 2000. Attenuation of apoptosis underlies B lymphocyte stimulator enhancement of humoral immune response. J. Exp. Med. 192:953-964.

20. Batten, M., et al. 2000. BAFF mediates survival of peripheral immature B lymphocytes. J. Exp. Med. 192:1453-1465.

21. Schiemann, B., et al. 2001. An essential role for BAFF in the normal development of B cells through a BCMA-independent pathway. Science. 293:2111-2114.

22. Gross, J.A., et al. 2001. TACI-Ig neutralizes molecules critical for B cell development and autoimmune disease: impaired B cell maturation in mice lacking BLyS. Immunity. 15:289-302.

23. Mackay, F., et al. 1999. Mice transgenic for BAFF develop lymphocytic disorders along with autoimmune manifestations. J. Exp. Med. 190:1697-1710.

24. Loder, F., et al. 1999. B cell development in the spleen takes place in discrete steps and is determined by the quality of B cell receptor-derived signals. J. Exp. Med. 190:75-89.

25. Martin, F., and Kearney, J.F. 2000. Positive selection from newly formed to marginal zone $\mathrm{B}$ cells depends on the rate of clonal production, CD19, and btk. Immunity. 12:39-49.

26. Zhang, J., et al. 2001. A role for B lymphocyte stimulator in systemic lupus erythematosus. J. Immunol. 166:6-10.

27. Cheema, G.S., Roschke, V., Hilbert, D.M., and Stohl, W. 2001. Elevated serum B lymphocyte stimulator levels in patients with systemic immunebased rheumatic diseases. Arthritis Rheum. 44:1313-1319.

28. Siegel, R.M., and Lenardo, M.J. 2001. To B or not to B: TNF family signalling in lymphocytes. Nat. Immunol. 2:577-578.

29. Gross, J.A., et al. 2000. TACI and BCMA are receptors for a TNF homologue implicated in B-cell autoimmune disease. Nature. 404:995-999.

30. Wang, H., et al. 2001. TACI-ligand interactions are required for T cell activation and collagen-induced arthritis in mice. Nat. Immunol. 2:632-637.

31. Schneider, P., et al. 1999. BAFF, a novel ligand of the tumor necrosis factor (TNF) family, stimulates B-cell growth. J. Exp. Med. 189:1747-1756.

32. White, S.C., and Casarett, G.W. 1974. Induction of experimental autoallergic sialadenitis. J. Immunol. 112:178-185.

33. Arnett, F.C., et al. 1987. The American Rheumatism Association 1987 revised criteria for the classification of rheumatoid arthritis. Arthritis Rheum. 31:315-324.

34. Tan, E.M., et al. 1982. The 1982 revised criteria for the classification of systemic lupus erythematosus. Arthritis Rheum. 25:1271-1277.

35. Vitali, C., et al. 1993. Preliminary criteria for the classification of Sjögren syndrome. Results of a prospective concerted action supported by the European Community. Arthritis Rheum. 36:340-347.

36. Chisholm, D.M., and Mason, D.K. 1968. Labial salivary gland biopsy in Sjögren's disease. J. Clin. Pathol. 21:656-660.

37. Kinoshita, G., et al. 1999. Molecular chaperones are targets of autoimmunity in Ro(SS-A) immune mice. Clin. Exp. Immunol. 115:268-274.

38. Wells, S.M., Kantor, A.B., and Stall, A.M. 1994. CD43 (S7) expression identifies peripheral B cell subsets. J. Immunol. 153:5503-5515.

39. Amano, M., et al. 1998. CD1 expression defines subsets of follicular and marginal zone B cells in the spleen: $\beta 2$-microglobulin-dependent and independent forms. J. Immunol. 161:1710-1717.

40. Khare, S.D., et al. 2000. Severe B cell hyperplasia and autoimmune disease in TALL-1 transgenic mice. Proc. Natl. Acad. Sci. USA. 97:3370-3375.

41. Robinson, C.P., et al. 1998. Transfer of human serum IgG to nonobese diabetic Ig $\mu^{\text {null }}$ mice reveals a role for autoantibodies in the loss of secretory function of exocrine tissue in Sjögren's syndrome. Proc. Natl. Acad. Sci. USA. 95:7538-7543.

42. Strasser, A., et al. 1991. Enforced BCL2 expression in B-lymphoid cells prolongs antibody responses and elicits autoimmune disease. Proc. Natl. Acad. Sci. USA. 88:8661-8665.

43. Bouillet, P., et al. 1999. Proapoptotic Bcl-2 relative Bim required for certain apoptotic responses, leukocyte homeostasis, and to preclude autoimmunity. Science. 286:1735-1738.

44. Zeng, D., Lee, M.-K., Tung, J., Brendolan, A., and Strober, S. 2000. A role for $\mathrm{CD} 1$ in the pathogenesis of lupus in NZB/NZW mice. J. Immunol. 164:5000-5004.

45. Grimaldi, C.M., Michael, D.J., and Diamond, B. 2001. Expansion and activation of a population of autoreactive marginal zone B cells in a model of estrogen-induced lupus. J. Immunol. 167:1886-1890.

46. Fagarasan, S., and Honjo, T. 2000. T-independent immune response: new aspects of B cell biology. Science. 290:89-92.

47. Anaya, J.M., McGuff, H.S., Banks, P.M., and Talal, N. 1996. Clinicopathological factors relating malignant lymphoma with Sjögren's syndrome. Semin. Arthritis Rheum. 25:337-346.

48. Martin, F., and Kearney, J.F. 2001. B1 cells: similarities and differences with other B cell subsets. Curr. Opin. Immunol. 13:195-201.

49. McCluskey, J., et al. 1998. Determinant spreading: lessons from animal models and human disease. Immunol. Rev. 164:209-229.

50. Thieblemont, C., Berger, F., and Coiffier, B. 1995. Mucosa-associated lymphoid tissue lymphomas. Curr. Opin. Oncol. 7:415-420. 